\title{
APLICAÇÃO DE POTÁSSIO, MAGNÉSIO E CALCÁRIO EM MUDAS DE BANANEIRA 'PRATA-ANÃ' (AAB) ${ }^{1}$
}

\author{
JOSÉ TADEU ALVES DA SILVA², INÊZ PEREIRA DA SILVA³ ${ }^{3}$ ASTHOR DE MOURA NETO³, ÉDIO LUIZ DA COSTA ${ }^{4}$
}

RESUMO-Para aplicar elevada quantidade de K no solo, é necessário que exista Mg em quantidade adequada, para evitar o aparecimento de distúrbios fisiológicos na bananeira. O objetivo deste trabalho foi avaliar os efeitos da aplicação de potássio (K), magnésio $(\mathrm{Mg})$ e calcário sobre o desenvolvimento de mudas de bananeira 'Prata-anã' (AAB) cultivadas em casa de vegetação. Os tratamentos foram distribuídos no delineamento de blocos casualizados, com quatro repetições em esquema fatorial $(5 \times 5 \times 2)$, correspondentes a cinco doses de $\mathrm{K}\left(0 ; 200 ; 400 ; 800\right.$ e $\left.1.600 \mathrm{mg} \mathrm{dm}^{-3}\right)$, cinco doses de $\mathrm{Mg}\left(0 ; 30 ; 60 ; 90\right.$ e $\left.180 \mathrm{mg} \mathrm{dm}^{-3}\right)$ e duas doses de calcário dolomítico $(0$ e $\left.2 \mathrm{t} \mathrm{ha}^{-1}\right)$. A produção da massa da matéria seca das mudas de bananeira aumentou com as aplicações de $\mathrm{K}$ e calcário no solo e reduziu com as doses de $\mathrm{Mg}$. Para a produção máxima da massa da matéria seca da parte aérea das mudas de bananeira, a relação Ca: $\mathrm{K}: \mathrm{Mg}$ no solo apresentou valores de 2,0: 0,3: 0,9, que correspondem a $62 \%, 9 \%$ e $28 \%$ da saturação por base, respectivamente.

Termos para Indexação: Musa sp., nutrição, solo.

\section{POTASSIUM, MAGNESIUM AND LIMESTONE APPLICATION IN BANANA TREE SEEDLINGS 'PRATA ANÃ' (AAB)}

\begin{abstract}
To apply high amount of $\mathrm{K}$ in the soil, it is necessary that Mg in adequate amount exists, in order to prevent the appearance of physiologic disturbances in banana tree. The objective of this work was to verify the effects of the application of $\mathrm{K}, \mathrm{Mg}$ and limestone in the development of banana tree seedlings 'Prata Anã'(AAB) cultivated in greenhouse. The treatments were distributed in a complete block design, with four repetitions, in factorial scheme $(5 \times 5 \times 2)$, corresponding to five $\mathrm{K}$ rates $(0,200,400$, 800 and $\left.1600 \mathrm{mg} \mathrm{dm}^{-3}\right)$, five $\mathrm{Mg}$ rates $\left(0,30,60,90\right.$ and $\left.180 \mathrm{mg} \mathrm{dm}^{-3}\right)$ and two limestone rates $\left(0\right.$ and $\left.2 \mathrm{t} \mathrm{ha}^{-1}\right)$. The dry matter production of banana tree seedlings increased with the applications of $\mathrm{K}$ and limestone in soil and reduced with the rates of Mg. For the maximum production of dry matter of the aerial part of the banana tree seedlings the relationships $\mathrm{Ca}: \mathrm{K}: \mathrm{Mg}$ in the soil presented values of 2,0: $0,3: 0,9$, that correspond to $62 \%, 9 \%$ and $28 \%$ of the saturation for base, respectively.
\end{abstract}

Index Terms: Musa sp., nutrition, soil.

\section{INTRODUÇÃO}

A adubação é um importante fator no sistema de produção da bananeira. Esta deve ser balanceada, para evitar que ocorram distúrbios nutricionais e, conseqüentemente, queda na produtividade. Assim, é necessário que no solo, onde se cultiva a bananeira, estabeleça-se um equilíbrio entre os nutrientes. $\mathrm{O}$ desbalanço entre $\mathrm{K} \mathrm{e} \mathrm{Mg}$ pode ocorrer com freqüência em virtude das elevadas quantidades de $\mathrm{K}$ aplicadas nos solos cultivados com bananeiras (Silva et al., 1999; Silva \& Carvalho, 2005).

A interação entre nutrientes em cultivos de bananeira tem sido bastante estudada. As interações mais estudadas são entre os cátions K, Ca e Mg (Borges, 2004). Para que se possa aplicar elevada quantidade de K no solo, é necessário que exista $\mathrm{Mg}$ em quantidades adequadas, a fim de evitar o aparecimento de distúrbios fisiológicos na bananeira (Silva et al., 1999). Delvaux (1995) relata que solos que apresentam relações $\mathrm{K} / \mathrm{Mg}$ entre 0,30 a 0,45 , encontram-se balanceados. Assim, relação superior a 0,6 indica excesso de $\mathrm{K}$ ou deficiência de $\mathrm{Mg}$, enquanto inferior a 0,2 mostra deficiência de $\mathrm{K}$ ou excesso de $\mathrm{Mg}$ no solo. De acordo com este autor, teores de K no solo, que variam de 200 a $350 \mathrm{mg} \mathrm{dm}^{-3}$, são normalmente suficientes para o crescimento e a produção da bananeira, entretanto, se o Ca e Mg estiverem presentes em quantidades elevadas, pode ocorrer deficiência de K. De acordo com Souza et al. (1999), é importante para bananeira não somente os teores absolutos de $\mathrm{K}, \mathrm{Ca}$ e $\mathrm{Mg}$ no solo, mas, principalmente, o equilíbrio entre eles.

$\mathrm{Na}$ avaliação do estado nutricional de bananeira 'Prataanã'(AAB) no norte de Minas Gerais, Silva \& Carvalho (2005) verificaram que os excessos de $\mathrm{Ca}, \mathrm{Mg}$ e $\mathrm{Mn}$, detectados pelo método DRIS, contribuíram para a deficiência de K em $23 \%$ dos bananais da região, apesar de o K ter sido aplicado periodicamente. Verificaram correlações negativas entre o teor de $\mathrm{K}$ foliar e os teores foliares de $\mathrm{Ca}, \mathrm{Mg}$ e $\mathrm{Mn}$.

O objetivo deste trabalho foi verificar os efeitos da aplicação de K, Mg e calcário sobre o desenvolvimento de mudas de bananeira 'Prata-anã' cultivadas em casa de vegetação.

${ }^{1}$ (Trabalho 183-07). Recebido em: 31-07-2007. Aceito para publicação em: 02-07-2008. Trabalho apresentado no XIX Congresso Brasileiro de Fruticultura.

${ }^{2}$ Pesquisador da EPAMIG/CTNM, DSc. Solos e Nutrição de Plantas, Caixa Postal 12, Nova Porteirinha-MG, e-mail: josetadeu@epamig.br

${ }^{3}$ Estudantes do curso de Agronomia da Universidade Estadual de Montes Claros-MG, e-mail: inezps@yahoo.com.br.

${ }^{4}$ Pesquisador da EPAMIG/CTNM, DSc. Engenharia Agrícola, Caixa Postal 12, Nova Porteirinha-MG, e-mail: edio.costa@epamig.br

Rev. Bras. Frutic., Jaboticabal - SP, v. 30, n. 3, p. 782-786, Setembro 2008 


\section{MATERIAL E MÉTODOS}

Foram coletadas amostras de Latossolo VermelhoAmarelo de textura média (LVA), na profundidade de $0-20 \mathrm{~cm}$. As amostras foram passadas em peneira de $4 \mathrm{~mm}$ de abertura, das quais foram retiradas subamostras que foram submetidas às análises químicas e granulométrica. Os resultados dessas análises são apresentados na Tabela 1.

Os tratamentos foram distribuídos no delineamento de blocos casualizados, com quatro repetições, em esquema fatorial $5 \times 5 \times 2$, correspondentes a cinco doses de $\mathrm{K}(0 ; 200 ; 400 ; 800$ e $\left.1.600 \mathrm{mg} \mathrm{dm}^{-3}\right)$, cinco doses de $\operatorname{Mg}\left(0 ; 30 ; 60 ; 90\right.$ e $\left.180 \mathrm{mg} \mathrm{dm}^{-3}\right) \mathrm{e}$ duas doses de calcário dolomítico $\left(0\right.$ e $\left.2 \mathrm{t} \mathrm{ha}^{-1}\right)$. As fontes de $\mathrm{K}$ e $\mathrm{Mg}$ utilizadas foram cloreto de potássio e sulfato de magnésio, respectivamente. Em todos os vasos, aplicaram-se nitrogênio (200 $\mathrm{mg} \mathrm{dm}^{-3} \mathrm{de}$ solo), fósforo ( $150 \mathrm{mg} \mathrm{dm}^{-3} \mathrm{de}$ solo) e uma mistura de micronutrientes em solução (em mg vaso ${ }^{-1}: 15,0$ de zinco; 15,0 de cobre e 15,0 de boro).

As mudas de bananeira obtidas de cultura de tecido foram cultivadas em vasos contendo $10 \mathrm{dm}^{-3}$ do solo previamente analisado. As mudas foram cultivadas durante 90 dias, e após esse período foram avaliadas a altura das plantas e o diâmetro do pseudocaule. As plantas foram cortadas rente ao solo, em seguida realizou-se a separação das raízes das plantas do solo de cada vaso. As raízes foram secas e pesadas, e a parte aérea foi seca, pesada, moída, mineralizada e analisada. A mineralização foi realizada por digestão-nitroperclórica. No extrato obtido, foram determinados os teores de $\mathrm{K}, \mathrm{Ca}$ e $\mathrm{Mg}$, conforme metodologia descrita em Malavolta et al. (1997).

Após as mudas de bananeira terem sido cortadas rente ao solo, amostras de solo foram retiradas em cada vaso, para determinar os teores de $\mathrm{K}, \mathrm{Ca}$ e $\mathrm{Mg}$ disponíveis, conforme Embrapa (1997).

Com os dados de desenvolvimento vegetativo das mudas, de produção da massa da matéria seca da parte aérea e das raízes das plantas, dos teores de $\mathrm{K}, \mathrm{Ca}$ e $\mathrm{Mg}$ do solo e da parte aérea das plantas, foram realizadas análises de variância e de regressão conforme Alvarez (1994).

\section{RESULTADOS E DISCUSSÃO}

A altura e o diâmetro do pseudocaule das mudas aumentaram de forma quadrática com as doses de $\mathrm{K}$ e diminuíram linearmente com as doses de $\mathrm{Mg}$, quando se aplicou calcário. Nos tratamentos onde não se aplicou calcário, a aplicação de $\mathrm{Mg}$ elevou de forma quadrática o diâmetro do pseudocaule e reduziu linearmente a altura das mudas (Tabela 2). A altura máxima $(43,0 \mathrm{~cm})$ e o diâmetro máximo do pseudocaule $(3,8 \mathrm{~cm})$ das mudas, nos tratamentos com aplicação de calcário, foram obtidos com aplicação de 350 e $437,5 \mathrm{mg} \mathrm{dm}^{-3}$ de K, respectivamente, sem aplicação de $\mathrm{Mg}$. Nos tratamentos sem aplicação de calcário, a altura máxima $(39,3 \mathrm{~cm})$ foi obtida com aplicações de 430 e $0 \mathrm{dm}^{-}$ ${ }^{3}$ de $\mathrm{K}$ e $\mathrm{Mg}$, respectivamente. O diâmetro máximo $(3,7 \mathrm{~cm})$ do pseudocaule das mudas foi obtido com aplicações de 222 e 85 $\mathrm{mg} \mathrm{dm}{ }^{-3}$ de $\mathrm{K} \mathrm{e} \mathrm{Mg}$, respectivamente (Tabela 2).

De acordo com o teste $t(P<0,01)$, verificou-se que a produção da massa da matéria seca da parte aérea das mudas, nos tratamentos com aplicação de calcário $(31,6 \mathrm{~g})$, foi significativamente maior em relação àquela onde não houve aplicação de calcário $(27,6 \mathrm{~g})$. Não houve efeitos significativos da aplicação de calcário, isoladamente, sobre a altura e o diâmetro do pseudocaule. Resultados semelhantes foram encontrados por Saes (1995) em mudas de bananeira Nanicão e Santos et al. (2004) em mudas de bananeira 'Prata-anã'.

Na presença ou na ausência de calcário, a aplicação de K proporcionou efeito quadrático sobre a produção da massa da matéria seca da parte aérea das mudas de bananeira. A aplicação de $\mathrm{Mg}$, na presença ou ausência de calcário, proporcionou efeito linear negativo (Figuras 1 e 2). Para os tratamentos em que não foi aplicado calcário, a produção máxima da massa da matéria seca da parte aérea $(32,1 \mathrm{~g})$ foi obtida com aplicação de 398 e 0 $\mathrm{mg} \mathrm{dm}{ }^{-3}$ de $\mathrm{K} \mathrm{e} \mathrm{Mg}$, respectivamente; para os tratamentos com aplicação de calcário, as doses para obter a produção máxima $(39,0 \mathrm{~g})$ foram de $458 \mathrm{e} 0 \mathrm{mg} \mathrm{dm}^{-3} \mathrm{de} \mathrm{K} \mathrm{e} \mathrm{Mg}$, respectivamente. $\mathrm{Na}$ ausência e na presença de calcário, os aumentos máximos na produção da massa da matéria seca da parte aérea, proporcionados pela aplicação de $\mathrm{K}$ no solo, foram de 4,9\% e $6,8 \%$, respectivamente. A aplicação de calcário potencializou o efeito do K sobre a produção de massa da matéria seca das mudas.

A aplicação de $\mathrm{K}$ proporcionou efeito quadrático sobre a produção da massa da matéria seca das raízes das mudas de bananeira, na presença ou na ausência de calcário (Figuras 3 e 4). As doses de $K$ para proporcionar a máxima produção da massa da matéria seca das raízes (18,5 e 51,2 g), foram de 225 e $523 \mathrm{mg}$ $\mathrm{dm}^{-3}$ para as plantas que não receberam e para as que receberam calcário, respectivamente. Nos tratamentos com ausência de calcário, o aumento na produção da massa da matéria seca das raízes foi de apenas $1,6 \%$, enquanto com a presença do calcário o aumento foi de $13 \%$. Em trabalho realizado por Santos et al. (2004), os autores verificaram efeitos quadráticos da aplicação de $\mathrm{KNO}_{3}$ sobre a altura, diâmetro do pseudocaule, número de folhas e área foliar de mudas de bananeira 'Prata-anã'.

De acordo com o teste $\mathrm{t}(\mathrm{P}<0,01)$, a produção da massa da matéria seca das raízes das mudas, nos tratamentos com aplicação de calcário $(31,8 \mathrm{~g})$, foi significativamente maior em relação àquela dos tratamentos em que não foi aplicado calcário $(16,2 \mathrm{~g})$. O Ca é o nutriente mais importante para o crescimento das raízes das plantas (Furtini et al., 2001).

$\mathrm{Na}$ ausência do calcário, não ocorreram efeitos significativos da aplicação de Mg sobre a produção da massa da matéria seca das raízes, entretanto, na presença do calcário, a aplicação de $\mathrm{Mg}$ reduziu de forma quadrática a produção da massa da matéria seca das raízes (Figuras 3 e 4). As aplicações do calcário e do $\mathrm{Mg}$ podem ter causado desequilíbrio nutricional, provocando redução na produção da massa da matéria seca das mudas.

Os teores de $\mathrm{Ca}$ e $\mathrm{Mg}$ no solo elevaram-se de 1,3 e 0,7 $\mathrm{cmol}_{\mathrm{c}} \mathrm{dm}^{-3}$ (Tabela 1) para 2,0 e $0,9 \mathrm{cmol}_{\mathrm{c}} \mathrm{dm}^{-3}$, respectivamente, após a aplicação do calcário dolomítico. $\mathrm{O}$ teor de $\mathrm{K}$ disponível antes da aplicação desse nutriente no solo foi de $0,08 \mathrm{cmol}_{\mathrm{c}} \mathrm{dm}^{-3}$. Equações de regressão foram ajustadas entre os teores de $\mathrm{K}, \mathrm{Ca}$ 
e Mg disponíveis com as doses de $\mathrm{K}$ e $\mathrm{Mg}$ aplicadas no solo (Tabela 3). Verificou-se que as aplicações de K e Mg no solo não influenciaram no teor de $\mathrm{Ca}$ disponível.

Foram ajustadas equações de regressão entre a altura, o diâmetro do pseudocaule, a produção da massa da matéria seca da parte aérea e das raízes das mudas de bananeira com os teores de K e Mg disponíveis no solo (Tabela 4). Nos tratamentos com aplicação de calcário, verificou-se que o teor de $\mathrm{K}$ disponível no solo ajustou-se de forma quadrática com os parâmetros de produção e desenvolvimento das mudas. Onde não houve aplicação de calcário, apenas a altura e a produção da massa da matéria seca da parte aérea relacionaram-se significativamente, de forma quadrática, com o teor de $\mathrm{K}$ disponível no solo (Tabela 4).

Nos tratamentos com aplicação de calcário, o teor de $\mathrm{Mg}$ disponível no solo relacionou-se negativamente, de forma linear, com a altura, o diâmetro do pseudocaule e a produção da massa da matéria seca da parte aérea e das raízes das mudas de bananeira. Quando não se aplicou calcário, o teor de Mg disponível no solo relacionou-se com a altura das plantas, significativamente, de forma quadrática e de forma linear negativa com a produção da massa da matéria seca das plantas (Tabela 4).

Os teores de $\mathrm{Ca}, \mathrm{K}$ e $\mathrm{Mg}$ disponíveis no solo, para obter a máxima produção da massa da matéria seca da parte aérea $(39,0$ g), foram de 2,0; 0,3 e 0,9 $\mathrm{cmol}_{\mathrm{c}} \mathrm{dm}^{-3}$, obtida nos tratamentos onde se aplicou calcário. Esses valores correspondem a $62 \%, 9 \%$ e $28 \%$ da saturação por bases, respectivamente. Para bananeira em fase de produção, Martin-Prével (1985) preconiza que, para alcançar alta produtividade, os valores de $\mathrm{Ca}: \mathrm{K}: \mathrm{Mg}$ no solo deva corresponder a $60-70 \%, 10 \%$ e $20-30 \%$ da saturação por bases.

$\mathrm{O}$ aumento das doses de $\mathrm{K}$ aplicadas no solo elevou, de forma quadrática, o teor de $\mathrm{K}$ na matéria seca da parte aérea das mudas (Tabela 5). Nos tratamentos sem aplicação de calcário, o aumento das doses de $\mathrm{Mg}$ reduziu linearmente o teor de $\mathrm{K}$. Ocorreu resposta quadrática negativa das doses de $\mathrm{K}$ e linear negativa das doses de $\mathrm{Mg}$ sobre o teor de Ca na parte aérea das mudas, nos tratamentos com aplicação de calcário; naqueles sem aplicação de calcário, as doses de $\mathrm{K}$ e Mg reduziram de forma quadrática o teor de Ca na parte aérea das mudas.

Nos tratamentos com aplicação de calcário; o teor de $\mathrm{Mg}$ na parte aérea das mudas elevou-se de forma linear e, nos tratamentos sem aplicação de calcário, o teor de Mg elevou-se de forma quadrática com o aumento das doses de $\mathrm{Mg}$ aplicadas no solo. Entretanto, o teor de Mg reduziu-se de forma quadrática com o aumento das doses de $\mathrm{K}$ aplicadas no solo com ou sem aplicação de calcário (Tabela 5). Essas regressões mostraram o antagonismo existente entre $\mathrm{K}, \mathrm{Ca}$ e $\mathrm{Mg}$.

As variáveis $\mathrm{K}$ e $\mathrm{Mg}$ nas equações de regressão dos tratamentos com aplicação de calcário, da Tabela 5, foram substituídas pelos seus respectivos valores ( 458 e $0 \mathrm{mg} \mathrm{dm}^{-3}$ ), os quais proporcionaram a máxima produção da massa da matéria seca da parte aérea das mudas. Obtiveram-se os valores de 4,4; 0,8 e 0,5 dag $\mathrm{kg}^{-1}$, para os teores dos nutrientes $\mathrm{K}, \mathrm{Ca}$ e $\mathrm{Mg}$, respectivamente, na parte aérea das mudas. As relações $\mathrm{K} / \mathrm{Ca}$, $\mathrm{K} / \mathrm{Mg}$ e $\mathrm{Ca} / \mathrm{Mg}$ encontradas foram de 5,5; 8,8 e 1,6, respectivamente. Segundo Borges (2004), a relação ideal entre $\mathrm{K} / \mathrm{Mg}$ nas folhas, no estágio de florescimento da planta, está entre o intervalo de 7 a 11.

TABELA 1 - Atributos químicos e físicos do solo. Nova Porteirinha. 2007

\begin{tabular}{|c|c|c|c|c|c|c|c|c|c|c|c|c|}
\hline Solos & $\mathrm{pH}$ & $\mathrm{P}^{1}$ & $\mathrm{~K}^{1}$ & $\mathrm{Ca}$ & $\mathrm{Mg}$ & CTC & $\mathrm{V}$ & P-rem ${ }^{2}$ & $\mathrm{MO}$ & Argila & Silte & Areia \\
\hline
\end{tabular}

${ }^{1}$ Extrator Mehlich $-1 \quad-{ }^{2}$ Fósforo remanescente

TABELA 2 - Desenvolvimento de mudas de bananeira em função de doses de $\mathrm{K}$ e Mg na presença e na ausência de calcário. Nova Porteirinha, 2007

\begin{tabular}{|c|c|c|}
\hline \multicolumn{3}{|c|}{ Com aplicação de calcário } \\
\hline Altura das mudas & $\hat{\mathrm{Y}}=42,1+3,5 \times 10^{-3} * * \mathrm{~K}-5,0 \times 10^{-6} * * \mathrm{~K}^{2}-4,9 \times 10^{-2} * * \mathrm{Mg}$ & $\mathrm{R}^{2}=0,953$ \\
\hline Diâmetro do pseudocaule & $\hat{\mathrm{Y}}=3,7+3,5 \times 10^{-} 4 * * \mathrm{~K}-4,0 \times 10^{-7} * * \mathrm{~K}^{2}-1,0 \times 10^{-2} * * \mathrm{Mg}$ & $\mathrm{R}^{2}=0,902$ \\
\hline \multicolumn{3}{|c|}{ Sem aplicação de calcário } \\
\hline Altura das mudas & $\hat{\mathrm{Y}}=38,4+4,3 \times 10^{-} 3^{*} * \mathrm{~K}-5,0 \times 10^{-6} * * \mathrm{~K}^{2}-1,3 \times 10^{-2} * * \mathrm{Mg}$ & $\mathrm{R}^{2}=0,932$ \\
\hline Diâmetro do pseudocaule & $\hat{\mathrm{Y}}=3,5+1,6 \times 10^{-4} * * \mathrm{~K}-3,6 \times 10^{-7} * * \mathrm{~K}^{2}+5,6 \times 10^{-3} * * \mathrm{Mg}-3,3 \times 10^{-5} * * \mathrm{Mg}^{2}$ & $\mathrm{R}^{2}=0,932$ \\
\hline
\end{tabular}

TABELA 3 - Equações de regressão ajustadas entre as doses de $\mathrm{K}$ e Mg aplicadas no solo e os teores de $\mathrm{K}$, Ca e Mg disponíveis. Nova Porteirinha, 2007

\begin{tabular}{|c|c|c|}
\hline \multicolumn{3}{|c|}{ Com aplicação de calcário } \\
\hline $\begin{array}{l}\text { K disponível }\left(\mathrm{mg} \mathrm{dm}^{-3}\right) \\
\text { Ca disponível }\left(\mathrm{cmol}_{\mathrm{c}} \mathrm{dm}^{-3}\right) \\
\text { Mg disponível }\left(\mathrm{cmol}_{\mathrm{c}} \mathrm{dm}^{-3}\right)\end{array}$ & $\begin{array}{l}\hat{\mathrm{Y}}=-33,9+3,0 \times 10^{-* *} * \mathrm{~K}+1,2 \times 10^{-4} * * \mathrm{~K}^{2}+0,13 \times 10^{1} * * \mathrm{Mg}-5,1 \times 10^{-3} * * \mathrm{Mg}^{2} \\
\hat{\mathrm{Y}}=\mathrm{ns} \\
\hat{\mathrm{Y}}=0,5+1,9 \times 10^{4} * * \mathrm{~K}+9,5 \times 10^{3} * * \mathrm{Mg}-3,0 \times 10^{-6} * * \mathrm{~K} \times \mathrm{Mg}\end{array}$ & $\begin{array}{r}\mathrm{R}^{2}=0,966 \\
\mathrm{R}^{2}=0,916\end{array}$ \\
\hline \multicolumn{3}{|c|}{ Sem aplicação de calcário } \\
\hline $\begin{array}{l}\text { K disponível }\left(\mathrm{mg} \mathrm{dm}^{-3}\right) \\
\text { Ca disponível }\left(\mathrm{cmol}_{\mathrm{c}} \mathrm{dm}^{-3}\right) \\
\text { Mg disponível }\left(\mathrm{cmol}_{\mathrm{cm}} \mathrm{dm}^{-3}\right)\end{array}$ & $\begin{array}{l}\hat{\mathrm{Y}}=15,9+4,4 \times 10^{-* * *} \mathrm{~K}+9,0 \times 10^{-5 * *} \mathrm{~K}^{2} \\
\hat{\mathrm{Y}}=\mathrm{ns} \\
\hat{\mathrm{Y}}=0\end{array}$ & $\begin{array}{l}\mathrm{R}^{2}=0,984 \\
\mathrm{R}^{2}=0,961\end{array}$ \\
\hline $\begin{array}{l}\text { Significativo a } 1 \% \text { de probabilidad } \\
\text { Significativo a } 5 \% \text { de probabilidad } \\
\text { Não-significativo }\end{array}$ & & \\
\hline
\end{tabular}

Rev. Bras. Frutic., Jaboticabal - SP, v. 30, n. 3, p. 782-786, Setembro 2008 
TABELA 4 - Equações de regressão ajustadas entre os teores de $\mathrm{K}$ e $\mathrm{Mg}$ disponíveis ( $\mathrm{cmol}_{\mathrm{c}} \mathrm{dm}^{-3}$ ) no solo e a altura (cm), diâmetro do pseudocaule $(\mathrm{cm})$, produção da massa da matéria seca da parte aérea e das raízes $(\mathrm{g})$ das mudas de bananeira. Nova Porteirinha, 2007

\begin{tabular}{|c|c|c|}
\hline \multicolumn{3}{|c|}{ Com aplicação de calcário } \\
\hline Altura das mudas & $\hat{\mathrm{Y}}=46,46+8,75 \times 10^{-1} * \mathrm{~K}-1,94 * \mathrm{~K}^{2}-4,87 * \mathrm{Mg}$ & $\mathrm{R}^{2}=0,718$ \\
\hline Diâmetro do pseudocaule & $\hat{\mathrm{Y}}=3,76+2,16 \times 10^{-1} * \mathrm{~K}-2,27 \times 10^{-1} * \mathrm{~K}^{2}-2,13 \times 10^{-1} * \mathrm{Mg}$ & $\mathrm{R}^{2}=0,719$ \\
\hline $\begin{array}{l}\text { Produção da massa da matéria } \\
\text { seca da parte aérea }\end{array}$ & $\hat{\mathrm{Y}}=42,08+5,07 * * \mathrm{~K}-7,62 * * \mathrm{~K}^{2}-4,31 * * \mathrm{Mg}$ & $\mathrm{R}^{2}=0,792$ \\
\hline $\begin{array}{l}\text { Produção da massa da matéria } \\
\text { seca das raízes }\end{array}$ & $\hat{\mathrm{Y}}=53,98+19,48^{* *} \mathrm{~K}-12,76^{* *} \mathrm{~K}^{2}-11,33^{* *} \mathrm{Mg}$ & $\mathrm{R}^{2}=0,848$ \\
\hline \multicolumn{3}{|c|}{ Sem aplicação de calcário } \\
\hline \multirow{4}{*}{$\begin{array}{l}\text { Altura das mudas } \\
\text { Diâmetro do pseudocaule } \\
\text { Produção da massa da matéria } \\
\text { seca da parte aérea } \\
\text { Produção da massa da matéria } \\
\text { seca das raízes }\end{array}$} & $\hat{\mathrm{Y}}=34,65+1,80 * \mathrm{~K}-1,96 * \mathrm{~K}^{2}+9,93 * \mathrm{Mg}-6,26 * \mathrm{Mg}^{2}$ & $\mathrm{R}^{2}=0,737$ \\
\hline & $\hat{\mathrm{Y}}=\mathrm{ns}$ & \\
\hline & $\hat{\mathrm{Y}}=32,42+3,74 * * \mathrm{~K}-3,16^{* *} \mathrm{~K}^{2}-3,67 * * \mathrm{Mg}$ & $\mathrm{R}^{2}=0,721$ \\
\hline & (1) & \\
\hline
\end{tabular}

** Significativo a $1 \%$ de probabilidade

* Significativo a $5 \%$ de probabilidade

ns Não-significativo

TABELA 5 Teores de K, Ca e Mg na parte aérea das mudas de bananeira em função de K e Mg aplicados em solo, na presença e na ausência de calcário. Nova Porteirinha, 2007

\begin{tabular}{|c|c|c|}
\hline $\begin{array}{l}\text { Teores de nutrientes } \\
\left(\mathrm{dag} \mathrm{kg}^{-1}\right)\end{array}$ & Com aplicação de calcário & \\
\hline $\mathrm{K}$ & $\hat{\mathrm{Y}}=2,5+5,2 \times 10^{-3} * * \mathrm{~K}-2,4 \times 10^{-6} * * \mathrm{~K}^{2}$ & $\mathrm{R}^{2}=0,672$ \\
\hline $\mathrm{Ca}$ & $\hat{\mathrm{Y}}=1,0-6,6 \times 10^{-4 * *} \mathrm{~K}+3,2 \times 10^{-7 * *} \mathrm{~K}^{2}-1,2 \times 10^{-3 * *} \mathrm{Mg}$ & $\mathrm{R}^{2}=0,827$ \\
\hline $\mathrm{Mg}$ & $\hat{\mathrm{Y}}=1,1-1,6 \times 10^{-3} * \mathrm{~K}+7,5 \times 10^{-7} * * \mathrm{~K}^{2}+6,6 \times 10^{-4} * \mathrm{Mg}$ & $\mathrm{R}^{2}=0,888$ \\
\hline \multicolumn{3}{|c|}{ Sem aplicação de calcário } \\
\hline $\mathrm{K}$ & $\hat{\mathrm{Y}}=3,5+5,9 \times 10^{-3} * * \mathrm{~K}-2,5 \times 10^{-6 * *} \mathrm{~K}^{2}-8,9 \times 10^{-3} * \mathrm{Mg} \quad \mathrm{R}^{2}=0,739$ & $\mathrm{R}^{2}=0,739$ \\
\hline $\mathrm{Ca}$ & $\hat{\mathrm{Y}}=0,8-4,5 \times 10^{-4} * * \mathrm{~K}+2,2 \times 10^{-7} * * \mathrm{~K}^{2}-3,7 \times 10^{-3} * * \mathrm{Mg}+1,1 \times 10^{-5 * *} \mathrm{Mg}^{2}$ & $\mathrm{R}^{2}=0,812$ \\
\hline $\mathrm{Mg}$ & $\hat{\mathrm{Y}}=0,5-5,8 \times 10^{-4} * * \mathrm{~K}+2,5 \times 10^{-7} * * \mathrm{~K}^{2}+1,8 \times 10^{-3} * * \mathrm{Mg}-4,9 \times 10^{-6 * *} \mathrm{Mg}^{2}$ & $\mathrm{R}^{2}=0,935$ \\
\hline
\end{tabular}

** Significativo a $1 \%$ de probabilidade

* Significativo a $5 \%$ de probabilidade

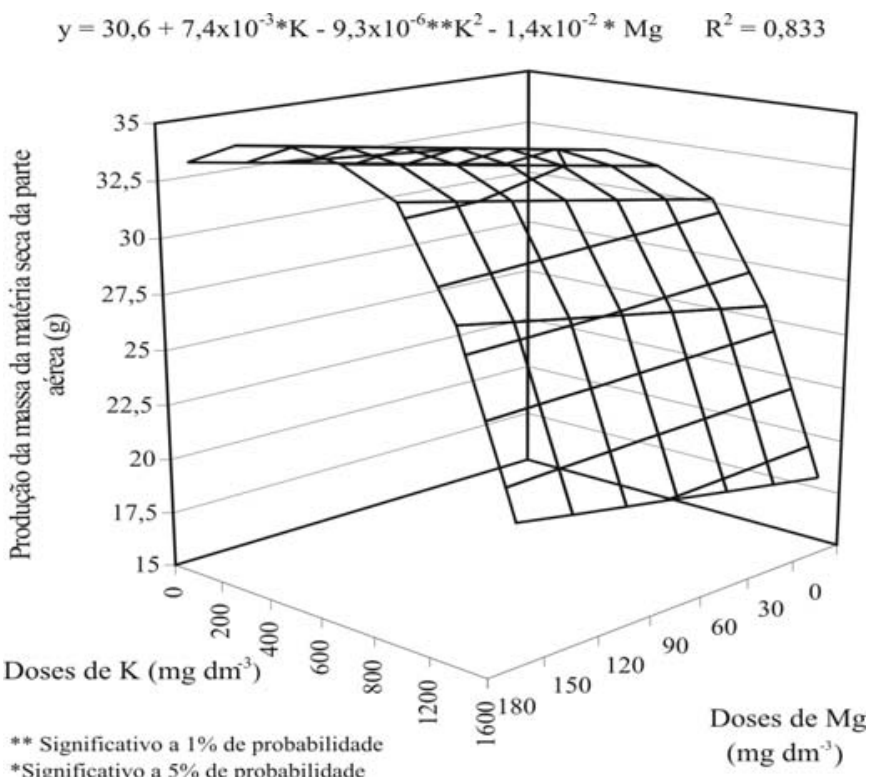

FIGURA 1 Produção da massa da matéria seca da parte aérea das mudas de bananeira em função das doses de $\mathrm{K} \mathrm{e} \mathrm{Mg}$ aplicadas no solo, na ausência de calcário. $\mathrm{y}=36,5+1,1 \times 10^{-2} * \mathrm{~K}-1,2 \times 10^{-5 * *} \mathrm{~K}^{2}-4,4 \times 10^{-2} * \mathrm{Mg} \quad \mathrm{R}^{2}=0,759$

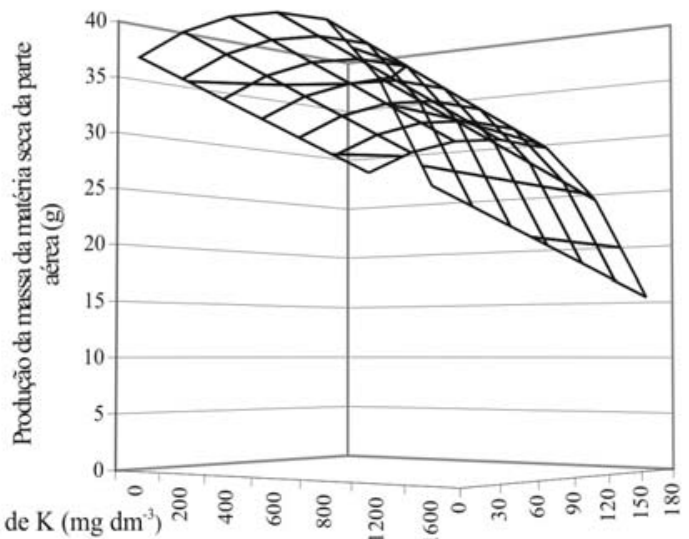

** Significativo a $1 \%$ de probabilidade

*Significativo a $5 \%$ de probabilidade

Doses de $\mathrm{Mg}\left(\mathrm{mg} \mathrm{dm}^{3}\right.$

FIGURA 2 - Produção da massa da matéria seca da parte aérea das mudas de bananeira em função das doses de K e Mg aplicadas no solo, na presença de calcário. 
$\mathrm{y}=18,2+2,7 \times 10-3^{* *} \mathrm{~K}-6,0 \times 10^{-6} * * \mathrm{~K}^{2} \quad \mathrm{R}^{2}=0,780$

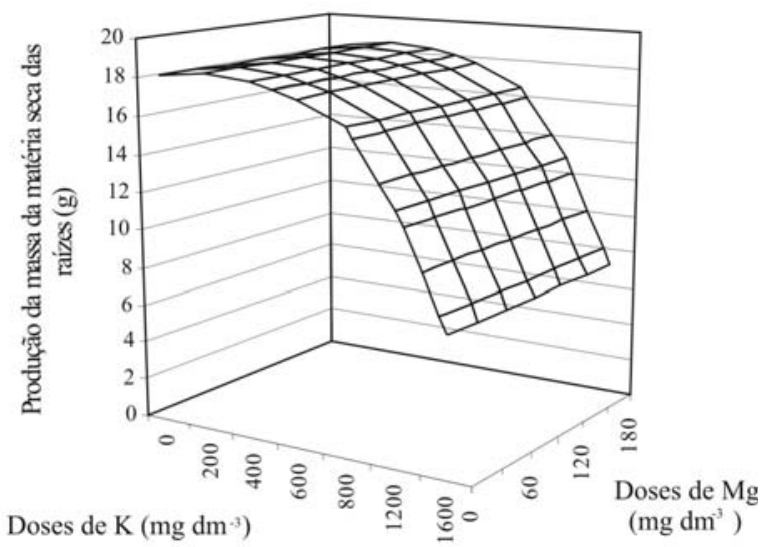

* Significativo a $1 \%$ de probabilidade

* Significativo a $5 \%$ de probabilidade

FIGURA 3 - Produção da massa da matéria seca das raízes das mudas de bananeira em função das doses de K e $\mathrm{Mg}$ aplicadas no solo, na ausência de calcário.

\section{CONCLUSÕES}

1-A produção da massa da matéria seca das mudas de bananeira aumentou com as aplicações de K e calcário no solo e reduziu com as doses de $\mathrm{Mg}$.

2-Para a produção máxima da massa da matéria seca da parte aérea das mudas de bananeira, a relação $\mathrm{Ca}: \mathrm{K}: \mathrm{Mg}$ no solo apresentou valores, em $\mathrm{cmol}_{\mathrm{c}} \mathrm{dm}^{-3}$, de 2,0: 0,3: 0,9, que correspondem a $62 \%, 9 \%$ e $28 \%$ da saturação por base, respectivamente.

\section{REFERÊNCIAS}

ALVAREZ V., V. H. Avaliação da fertilidade do solo. Viçosa: UFV, $1994.75 \mathrm{p}$.

BORGES, A. L. Interação entre nutrientes em bananeira. Cruz das Almas: Embrapa CNPMF, 2004. 2 p. (Banana em Foco, 55)

DELVAUX, B. Soil. In: GOWEN, S. Bananas and plantains. New York: Chapman \& Hall, 1995. p. 230-257.

EMBRAPA.Centro Nacional de Pesquisa de solos. Manual de métodos de análise de solo. 2.ed. revisado e atualizado. Rio de Janeiro: Centro Nacional de Pesquisa de Solos, 1997. 212p.

FURTINI NETO, A. E.; VALE, F. R. do; RESENDE, A. V. de; GUILHERME, L. R. G.; GUEDES, G. A. A. Fertilidade do solo. Lavras: UFLA/FAEPE, 2001.252p.

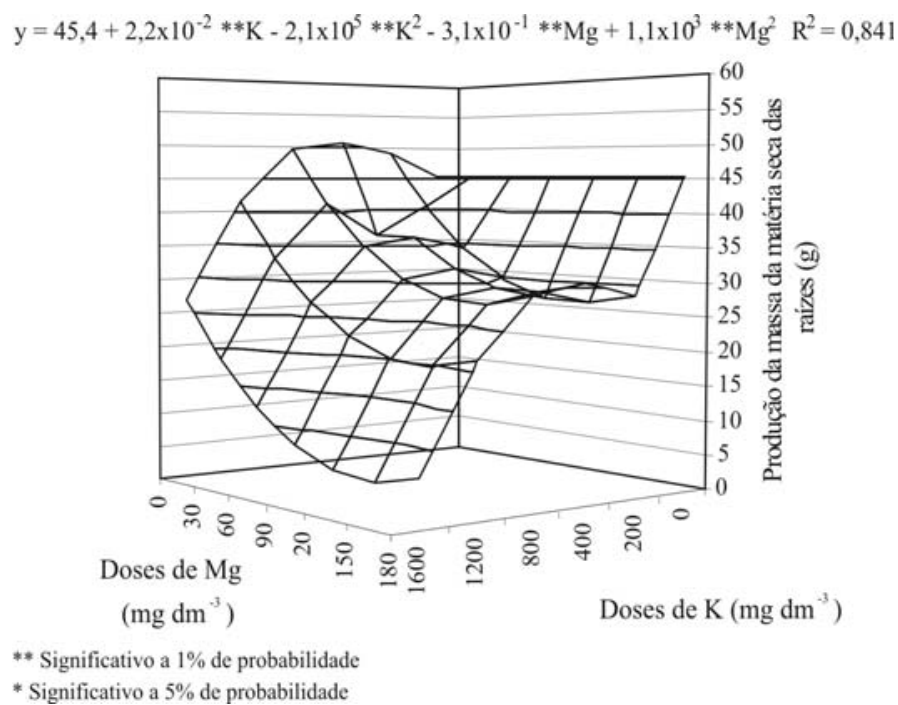

FIGURA 4 - Produção da massa da matéria seca das raízes das mudas de bananeira em função das doses de $\mathrm{K}$ e $\mathrm{Mg}$ aplicadas no solo, na presença de calcário.

MALAVOLTA, E.; VITTI, G. C.; OLIVEIRA, S. A. Avaliação do estado nutricional das plantas: princípios e aplicações. 2.ed. Piracicaba: POTAFÓS, 1997. 201 p.

MARTIN-PRÉVEL, P. Exigências nutricionais da bananicultura. In: SIMPÓSIO BRASILEIRO SOBRE BANANICULTURA, 1. 1984, Jaboticabal. Anais... Jaboticabal: UNESP/FCAV, 1985, p. 118-134.

SAES, L. A. Resposta da bananeira 'Nanicão' à calagem na região do vale do Ribeira. 1995. 82 f. Dissertação (Mestrado em Fitotecnia)- Escola Superior de Agricultural Luiz de Queiroz, Universidade de São Paulo, Piracicaba, 1995.

SANTOS, J. A.; SILVA, C. R. R.; CARVALHO, J.G.; NASCIMENTO, T. B. Efeito do calcário dolomítico e nitrato de potássio no desenvolvimento inicial de mudas da bananeira 'Prata anã' (AAB), provenientes de cultura in vitro. Revista Brasileira de fruticultura, Jaboticabal, v. 26, n. 1, p. 150-154, 2004.

SILVA, J. T. A. ; BORGES A. L., MALBURG, J. L. Solos, adubação e nutrição da bananeira. Informe agropecuário, Belo Horizonte, v. 20, n. 196, p. 21-36, 1999.

SILVA, J. T. A.; CARVALHO, J. G. Avaliação nutricional de bananeira Prata-Anã (AAB), sob irrigação no semi-árido do norte de Minas Gerais, pelo método Dris. Ciência e Agrotecnologia, Lavras, v. 29, n. 4, p. 731-739, 2005.

SOUZA, L.; BORGES, L. A.; SILVA, J. T. A. da. Características físicas e químicas de solos cultivados com bananeira, sob irrigação, na região norte de Minas Gerais. Cruz das AlmasBA: Embrapa Mandioca e Fruticultura, 1999. 24p. (Boletim de Pesquisa, 14). 